\title{
An Orthogonal Wavelet Transform Multi-modulus Blind Equalization Algorithm Based on Tabu Search DNA Genetic Optimization Algorithm
}

\author{
Guo Yecai $^{1,2, a}$, Lu Lu' ${ }^{1}$, Zhang Binglong ${ }^{1}$ \\ ${ }^{1}$ College of Electronic and Information Engineering, Nanjing University of Information Science and \\ Technology, Nanjing 210044, China \\ ${ }^{2}$ Jiangsu Collaborative Innovation Center on Atmospheric Environment and Equipment \\ Technology (CICAEET), Nanjing 210044,China \\ aemail: guo-yecai@163.com
}

Keywords: WTMMA; double-stranded DNA; tabu search strategy; adaptive probability; convergence rate; mean square error

\begin{abstract}
For the disadvantages of orthogonal wavelet transform multi-modulus blind equalization algorithm(WTMMA), such as slow convergence rate, large mean square error, and immerging in partial minimum easily, an orthogonal wavelet transform multi-modulus blind equalization algorithm based on Tabu search DNA genetic optimization algorithm(DNAGA-WTMMA) was proposed. Firstly, DNA populations were initialized by using double-stranded DNA form to select the single-stranded DNA sequences as the representative strands of individuals. Secondly, Tabu search strategy was introduced into crossover operations to avoid circuitous search, ensure the searching different paths, and escape from local optimum. Thirdly, in order to speed up the convergence rate and avoid premature convergence, the probability of crossover operation was decided by dynamic adaptive probability based on population prematurity degree index. Computer simulations show that, compared with multi-modulus blind equalization algorithm(MMA) and WTMMA, the proposed algorithm has the fastest convergence rate and the smallest mean square error.
\end{abstract}

\section{Introduction}

In wireless communication, blind equalization technology without sending the training sequence is one of important methods of suppressing the inter-symbol interference(ISI) caused by complicated communication channel and limited bandwidth. In the blind equalization algorithms, the constant modulus blind equalization algorithm(CMA) has good equalization performance for constant modulus signals, but it has large mean square error for high-order QAM signals. While the multi-modulus equalization algorithm(MMA) with the amplitude and phase information of output signals can reduce effectively the mean square error(MSE). Orthogonal wavelet multi-modulus blind equalization algorithm outperforms MMA in equalizing multi-modulus signals. However, the weight vector of the CMA and the MMA is updated by gradient search method, therefore, they are easy to fall into local convergence and difficult to obtain the global optimal solution[1].

Genetic algorithm(GA) has stronger robustness and global random search abilities and can find global optimal solution in complex, multi-peak large solution space. However, the traditional genetic algorithm is easy to fall into premature convergence and its convergence speed is slow[2]. DNA computing proposed by Adleman in 1994, has strong ability of parallel computing, can be used to search all potential solutions. Due to the nature connection with DNA computing and genetic algorithm, DNA computing is introduced into genetic algorithm to improve the performance of GA[3][4]5][6]. Tabu search algorithm(TS) is a global search algorithm with short-time memory, a tabu list (movements in the list were temporarily forbidden) and an aspiration criterion changing the content of tabu list are adopted[7][8]. Therefore, we introduce tabu search algorithm into DNA genetic algorithm to improve the performance of DNA genetic algorithm.

Based on the above analysis, an orthogonal wavelet transform multi-modulus blind equalization algorithm based on tabu search DNA genetic optimization algorithm(DNAGA-WTMMA) is 
proposed. This proposed algorithm uses cost function of the WTMMA as the fitness function of DNA-GA, then uses DNA-GA to solve the cost function of the equalizer to find optimal individual. Compared with multi-modulus blind equalization algorithm(MMA) and orthogonal wavelet transform multi-modulus blind equalization algorithm(WTMMA), the proposed algorithm has the fastest convergence rate and the smallest mean square error.

\section{Orthogonal Wavelet Transform Multi-modulus Blind Equalization Algorithm}

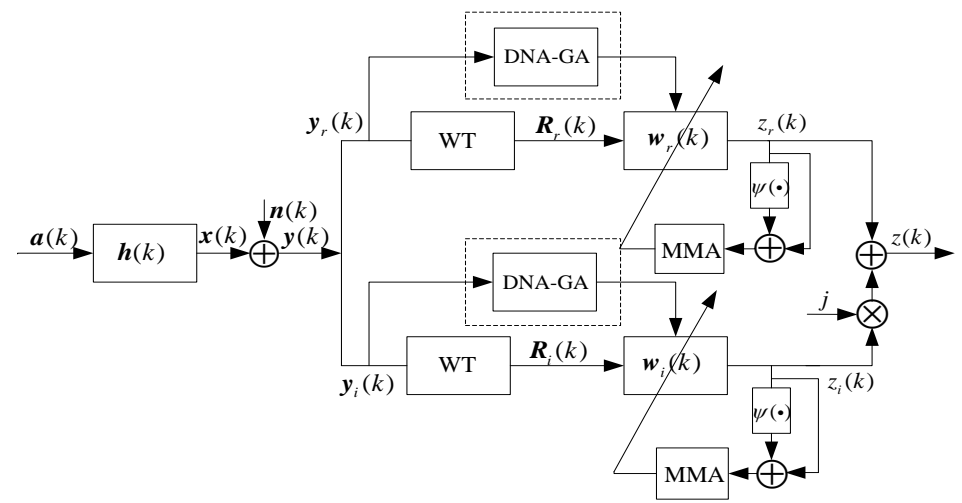

Fig.1. WTCMA based on DNA-GA

First, orthogonal wavelet transform multi-modulus blind equalization algorithm(WTCMA)[9] is shown in Figure 1. In Fig.1, $\boldsymbol{a}(k)=\boldsymbol{a}_{r}(k)+\mathrm{j} \boldsymbol{a}_{i}(k)$ is the transmitting signal, where $\boldsymbol{a}_{r}(k)$ and $\boldsymbol{a}_{i}(k)$ are real part and imaginary part of $\boldsymbol{a}(k) . \boldsymbol{h}(k)$ is the channel impulse response vector, $\boldsymbol{y}(k)=\boldsymbol{y}_{r}(k)+\mathrm{j}_{i}(k)$ is the input signal of equalizer, $\boldsymbol{R}_{r}(k)$ and $\boldsymbol{R}_{i}(k)$ are the orthogonal wavelet transform of signal vector $\boldsymbol{y}_{r}(k)$ and $\boldsymbol{y}_{i}(k), \boldsymbol{n}(k)$ is additive white Gaussian noise. $\boldsymbol{w}(k)=\boldsymbol{w}_{r}(k)+\mathrm{j} \boldsymbol{w}_{i}(k)$ is the weight vector of equalizer, $z(k)=z_{r}(k)+\mathrm{j} z_{i}(k)$ is the output signal of equalizer. From the wavelet analysis theory, the equalizer weight vector $\boldsymbol{w}(k)$ can be represented by a set of wavelet basis function,

$$
\begin{aligned}
& \boldsymbol{w}_{r}(k)=\sum_{j=1}^{J} \sum_{m=0}^{k_{j}} d_{r j, m} \bullet \varphi_{j, m}(k)+\sum_{m=0}^{k_{J}} v_{r J, m} \bullet \phi_{J, m}(k) \\
& \boldsymbol{w}_{i}(k)=\sum_{j=1}^{J} \sum_{m=0}^{k_{j}} d_{i j, m} \bullet \varphi_{j, m}(k)+\sum_{m=0}^{k_{J}} v_{i J, m} \bullet \phi_{J, m}(k)
\end{aligned}
$$

where $k=0,1, \cdots, L-1, L=2^{J}, \quad J$ is the biggest scale wavelet decomposition, $k_{j}=L / 2^{j}-1$ $(j=1,2, \cdots, J)$ is the biggest translation of the wavelet function, $\varphi_{j, m}(k)$ and $\phi_{J, m}(k)$ are wavelet function and scale function, $d_{r j, m}$ and $v_{r J, m}$ are real part of equalizer, $d_{i j, m}$ and $v_{i J, m}$ are imaginary part of equalizer.

After being transformed by orthogonal wavelet, $\boldsymbol{R}(k)$ can be represented as

$$
\boldsymbol{R}(k)=\boldsymbol{R}_{r}(k)+\mathrm{j} \boldsymbol{R}_{i}(k)=\boldsymbol{Q} \boldsymbol{y}_{r}(k)+\mathrm{j}\left(\boldsymbol{Q} \boldsymbol{y}_{i}(k)\right)
$$

where $\boldsymbol{Q}$ is orthogonal wavelet transform matrix, $\boldsymbol{R}_{r}(k)$ and $\boldsymbol{R}_{i}(k)$ can be represented as

$$
\begin{aligned}
& \boldsymbol{R}_{r}(k)=\left[u_{r 1,0}(k), u_{r 1,1}(k), \cdots, u_{r J, k_{J}}(k), s_{r J, 0}(k), \cdots, s_{r J, k_{J}}(k)\right]^{\mathrm{T}} \\
& u_{r j, m}(k)=\sum_{l=0}^{L-1} y_{r}(k-l) \varphi_{j, m}(l), s_{r J, m}(k)=\sum_{l=0}^{L-1} y_{r}(k-l) \varphi_{J, m}(l) \\
& \boldsymbol{R}_{i}(k)=\left[u_{i 1,0}(k), u_{i 1,1}(k), \cdots, u_{i J, k_{J}}(k), s_{i J, 0}(k), \cdots, s_{i J, k_{J}}(k)\right]^{\mathrm{T}} \\
& u_{i j, m}(k)=\sum_{l=0}^{L-1} y_{i}(k-l) \varphi_{j, m}(l), s_{i J, m}(k)=\sum_{l=0}^{L-1} y_{i}(k-l) \varphi_{J, m}(l)
\end{aligned}
$$

where $u_{j, m}(k)$ and $s_{J, m}(k)$ are wavelet function and scale transform coefficient. 
The output of the equalizer is written as

$$
z(k)=z_{r}(k)+\mathrm{jz} z_{i}(k)=\boldsymbol{w}_{r}^{\mathrm{T}}(k) \boldsymbol{R}_{r}(k)+\mathrm{j}\left(\boldsymbol{w}_{i}^{\mathrm{T}}(k) \boldsymbol{R}_{i}(k)\right)
$$

where $\boldsymbol{w}_{r}(k)$ and $\boldsymbol{w}_{i}(k)$ are real part and imaginary part of equalizer.

The cost function of the WTMMA can be defined as

$$
J_{\text {WTMMA }}=E\left\{\left(z_{r}^{2}(k)-R_{r W T M M A}^{2}\right)^{2}+\left(z_{i}^{2}(k)-R_{i \text { WTMMA }}^{2}\right)^{2}\right\}
$$

where $R_{r \text { WTMMA }}^{2}=E\left(a_{r}^{4}(k)\right) / E\left(a_{r}^{2}(k)\right)$ and $R_{\mathrm{iWTMMA}}^{2}=E\left(a_{i}^{4}(k)\right) / E\left(a_{i}^{2}(k)\right)$ represent real part and imaginary part modulus value.

The error function is denoted as

$$
e_{r \text { WTMMA }}(k)=z_{r}(n)\left(z_{r}^{2}(k)-R_{r W T M M A}^{2}\right), e_{\text {iWTMMA }}(k)=z_{i}(n)\left(z_{i}^{2}(k)-R_{\text {iWTMMA }}^{2}\right)
$$

The weight vector is updated by

$$
\boldsymbol{w}_{r}(k+1)=\boldsymbol{w}_{r}(k)-\mu \hat{\boldsymbol{R}}^{-1}(k) e_{r \mathrm{WTMMA}}(k) \boldsymbol{R}_{r}^{*}(k), \quad \boldsymbol{w}_{i}(k+1)=\boldsymbol{w}_{i}(k)-\mu \hat{\boldsymbol{R}}^{-1}(k) e_{\mathrm{iWTMMA}}(k) \boldsymbol{R}_{i}^{*}(k)
$$

where

$$
\hat{\boldsymbol{R}}^{-1}(k)=\operatorname{diag}\left[\sigma_{j, 0}^{2}(k), \sigma_{j, 1}^{2}(k), \cdots, \sigma_{J, k_{J}-1}^{2}(k), \sigma_{J+1,0}^{2}(k), \cdots, \sigma_{J+1, k_{J}-1}^{2}(k)\right]
$$

$\sigma_{j, k}^{2}(k)$ and $\sigma_{J+1, k_{j}}^{2}(k)$ is estimate of wavelet transform coefficient and scale transform coefficient, can be obtained by following formula:

$$
\sigma_{j, m}^{2}(k+1)=\beta \sigma_{j, m}^{2}(k)+(1-\beta)\left|u_{j, m}(k)\right|^{2}, \sigma_{J+1, m}^{2}(k+1)=\beta \sigma_{J+1, m}^{2}(k)+(1-\beta)\left|s_{J, m}(k)\right|^{2}
$$

where $0<\beta<1$ is smoothing factor.

\section{WTMMA Based on Tabu Search DNA Genetic Optimization Algorithm}

DNA coding. DNA molecule is composed of adenine(A), guanine( $\mathrm{G})$, cytosine $(\mathrm{C})$, and thymine(T). Therefore, equalizer weight vector will be coded by these four bases. In order to facilitate computer processing, we use integer 0,1,2,3 to denote these bases, for example, A,B,C,and D denote $0,1,2$, and 3,respectively. Meanwhile, the digital coding of bases must obey the matching rule between complementary base pair. So, DNA molecular can be represented by the number of quaternary.

Tabu crossover operation. The tabu search strategy is introduced into the crossover operation of GA to tabu crossover operation. Tabu crossover operation uses tabu list to record the fitness of individuals in population. Firstly, the "best so far" state is denoted by $S_{\text {best_so_far }}$, and it is initialized by mean fitness of parent population. The procedure of tabu crossover operation can be illustrated as follows ( $S_{p}^{1}$ and $S_{p}^{2}$ represent individual in population).

Begin

Parents $S_{p}^{1}, S_{p}^{2}$

$S_{\text {best_so } f a r}=$ the mean fitness of parent population

If Fitness $\left(S_{p}^{1}\right)>$ Fitness $\left(S_{p}^{2}\right) \quad S_{p}^{\max }=S_{p}^{1}$;

Else

$$
\left\{S_{p}^{\max }=S_{p}^{2}\right.
$$

parents $S_{p}^{1}, S_{p}^{2}$ generate offspring $S_{p+1}^{1}, S_{p+1}^{2}$ after crossover operation;

$$
\left.T\left(S_{p}^{1}, S_{p+1}^{1}, S_{p}^{\max }\right) ; T\left(S_{p}^{2}, S_{p+1}^{2}, S_{p}^{\max }\right) ;\right\}
$$

End

Function $T\left(S_{p}^{1}, S_{p+1}^{1}, S_{p}^{\max }\right)$

If Fitness $\left(S_{p+1}^{1}\right)>S_{\text {best_so_far }}$

$\left\{\right.$ offspring $S_{p+1}^{1}$ takes place of parent $S_{p}^{1}$;

Put Fitness $\left(S_{p+1}^{1}\right)$ into tabu list; 
$S_{\text {best_so_far }}=$ Fitness $\left(S_{p+1}^{1}\right)$; $\}$

Else if Fitness $\left(S_{p+1}^{1}\right)$ in tabu list

$\left\{\right.$ put $S_{p}^{\max }$ into next generation;

Else offspring $S_{p+1}^{1}$ takes place of parent $S_{p}^{1}$;

Put Fitness $\left(S_{p+1}^{1}\right)$ into tabu list; $\}$

End

$T\left(S_{p}^{2}, S_{p+1}^{2}, S_{p}^{\max }\right)$ is same with $T\left(S_{p}^{1}, S_{p+1}^{1}, S_{p}^{\max }\right)$.

There are three different crossover operation: replacement crossover operation, translocation crossover operation and reconstruction crossover operation.

Replacement crossover operation is shown in Figure 2(a). A pair of parents can be obtained by choosing randomly two individuals from the population, then some bases, whose quantity and position is same for two parents, are selected randomly. Then, two individuals exchange the selected parts to produce two new individuals.

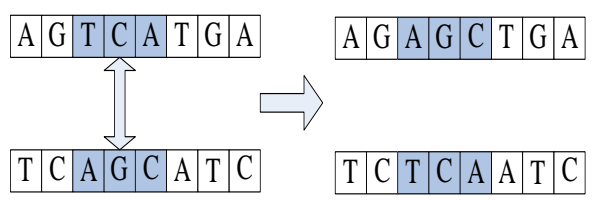

(a) Replacement

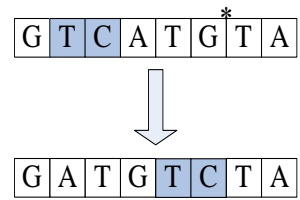

(b) Translocation

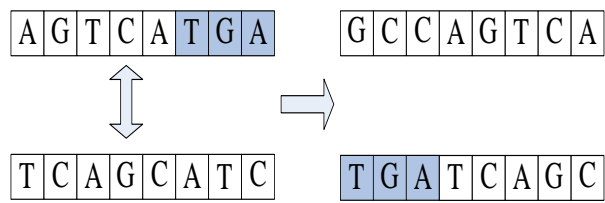

(c) Reconstruction

Fig.2. Crossover operations

Translocation crossover operation is shown in Figure 2(b). First, one individual from the population and one part in this individual are randomly selected, then this part moves to another location in this individual to produce a new individual.

Reconstruction crossover operation is shown in Figure 2(c). A pair of parents can be obtained by choosing randomly two individuals and marked as $A^{\prime}$ and $B^{\prime}$. We cut one part from the rear of parent A', and then this part is added to the front of parent B'. Since the length of the individual is fixed in DNA-GA, one part with the same length of the cut one is randomly generated and is added to the head of parent A', while the same length part is tailored from the rear of parent B'.

Mutation crossover operation occurs among all the individuals in the current population to generate various individuals. According to the principle of biology, there exist "cold spots" and "hot spots" in different locations of DNA sequence. We define the left half of the nucleotide bases of DNA sequence as the high bit position and the right half as the low bit position. In order to improve the global search ability at the beginning stage of evolution, we set a larger mutation probability for the high bit position. In order to obtain the global optimal solution and make the search of local optimal solutions be more accurate, the mutation probability in the high bit position is required to be reduced and the probability in the low bit position is required to be increased over generations. Hence, at the ending stage of evolution, the low bit position with a larger mutation probability enables the algorithm to find an accurate optimum solution. The adaptive mutation probabilities $p_{m h}$ and $p_{m l}$ are given by

$$
p_{m h}=a_{1}+\frac{b_{1}}{1+\exp \left[a\left(g-g_{0}\right)\right]}, \quad p_{m l}=a_{1}+\frac{b_{1}}{1+\exp \left[-a\left(g-g_{0}\right)\right]}
$$

where $p_{m h}$ and $p_{m l}$ represent the mutation probability of the high bit position and the low bit position, respectively. Coefficient $a_{1}$ denotes the initial mutation probability, coefficient $b_{1}$ is the range of the mutation probability. Coefficient $g_{0}$ is the turning point of hot spots and cold spots, and $a$ is the rate of change.

Selection operation. The individuals for the next generation is also an important process. In this paper, tournament selection is used, in which two individuals are compared against each other and the one with higher fitness value advances into the next generation. Note that elitism is also used in conjunction with tournament selection in attempt to guarantee the best individual is replicated into 
the next generation.

Adaptive crossover probability. Premature convergence must be considered in DNA genetic algorithm, because the premature convergence of the GA tends to make the population diversity decline. The updating speed of population is determined by crossover operation probability. The crossover operation probability is adjusted by population fitness value to improve the performance of DNA genetic algorithm.

We define that $F_{n}^{i}$ is the fitness value of the $i$ th individual, $F_{n_{-} \max }$ is the fitness value of the best individual in population, $\bar{F}_{n}$ is the average fitness value of population. $\bar{F}_{n_{-} \text {big }}$ is the average fitness value of individuals whose fitness values are higher than the average fitness value. Therefore, we define $\hat{F}_{n}^{\alpha}$ as follows

$$
\hat{F}_{n}^{\alpha}= \begin{cases}F_{n}^{\left(\left\lceil N_{\alpha}\right\rceil+1\right.}, & N_{\alpha} \notin Z \\ \frac{\left(F_{n}^{N_{\alpha}}+F_{n}^{\left(N_{\alpha}+1\right)}\right)}{2} & N_{\alpha} \in Z\end{cases}
$$

where $0<\alpha<1, N_{\alpha}=\alpha N,\lceil\bullet\rceil$ denotes roundup function. From above, we can define $\bar{F}_{n_{-} a v e}^{3^{\prime}}$ as follow:

$$
\bar{F}_{n_{-} \text {ave }}^{3^{\prime}}=\frac{1}{4} \hat{F}_{n}^{0.25}+\frac{1}{2} \hat{F}_{n}^{0.5}+\frac{1}{4} \hat{F}_{n}^{0.75}
$$

We define the premature degree index of population $P_{r}$ as follow:

$$
P_{r}=\left(F_{n_{-} \max }-\bar{F}_{n_{-} b i g}^{3^{\prime}}\right) / F_{n_{-} \max }
$$

where $\bar{F}_{n_{-} b i g}^{3^{\prime}}$ is the average fitness value of individuals whose fitness values are higher than $\bar{F}_{n_{-} a v e}^{3^{\prime}}$. The probability of DNA genetic algorithm is decided by the premature degree index of population, thus, we can obtain basic crossover operation probability as follow:

$$
P_{c}=\left(1+\exp \left(-\tau_{c} P_{r}\right)\right)^{-1}, \tau_{c}>0
$$

where $\tau_{c}=2.2$. From above, we can obtain replacement crossover operation probability, translocation crossover operation, and reconstruction crossover operation probability

$$
P_{1}=0.75 P_{c}, \quad P_{2}=0.5 P_{c}, \quad P_{3}=0.25 P_{c}
$$

The procedure of DNAGA-WTMMA. Based on the encoding and operations mentioned as above, we can summarize the frame of the proposed algorithm as follows:

Step1: Determine the fitness function. The objective function of DNA-GA is the cost function of WTMMA. In order to obtain the minimum value of the objective function, we define the fitness function $\operatorname{Fit}\left(\boldsymbol{w}_{m}\right)=b / J_{\text {WTMMA }}\left(\boldsymbol{w}_{m}\right), b$ is constant.

Step2:Initialize population according to corresponding coding method. Firstly, the equalizer weight vectors are denoted by double-stranded DNA molecule. Then selecting single-stranded DNA sequence whose fitness value is higher between two single-strand DNA sequence to represent the individual. Then, high-quality population and inferior population are divided into two groups according to individual fitness value. The best individuals are duplicated into next generation.

Step3:Execute the tabu crossover operation. We set up tabu list and initialize $S_{\text {best_so_far }}$. Two individuals from the population are selected randomly, and replacement crossover operation and translocation crossover operation are executed according to corresponding probability. If both replacement crossover operation and translocation crossover operation aren't executed, reconstruction crossover operation is executed according to probability. Those operations are repeatedly carried out to create N/2 new individuals. Finally, those new individuals are put into population, we will get 3N/2 individuals.

Step4: Mutation operation for each individual. For each individual, if the mutation occurs, the original individual is replaced with the mutated one. After carrying out mutation operation, tournament selection operations are executed to create M-1 new individuals. Those new individuals and elite form new population. Finally, the corresponding complementary single-strand DNA sequence is generated and the fitness value of all individuals is computed. 
Step5: Judge with the genetic interrupts condition. If the condition of maximum evolution generation is met, the individuals with largest fitness value from all the single-strand DNA molecules are selected as the best individuals. Otherwise, we repeat from step 2 to 4 until that the stop condition is met. Finally, the optimal solution will be obtained by decoding those best individuals, and then it is used to initialize the equalizer weight vector.

\section{Simulation Experiments}

In order to verity the effectiveness of the proposed algorithm, simulation experiments were completed and compared with the MMA and WTMMA. $\boldsymbol{h}(k)=[0.3132,-0.1040,0.8908,0.3134]$, signal to noise ratio(SNR) was $25 \mathrm{~dB}$, the length of equalizer was 16 . For 64QAM signal, DB2 orthogonal wavelet transform was used. Power initial value was set to 4 and forgetting factor $\beta$ was 0.99 . The step-size of the MMA was $\mu_{\text {MMA }}=0.6 \times 10^{-5}$, step-size of WTMMA was $\mu_{\text {WTMMA }}=1 \times 10^{-5}$, and step-size of the DNA-GA-WTMMA is $\mu_{\text {DNAGA-WTMmA }}=1.5 \times 10^{-6}$. The simulation results were shown in Figure 3.

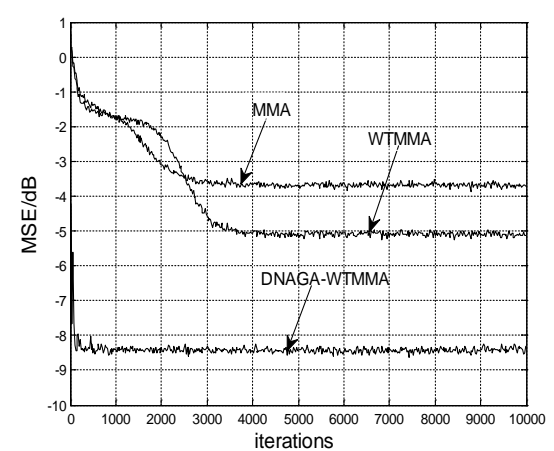

(a) The curves of MSE

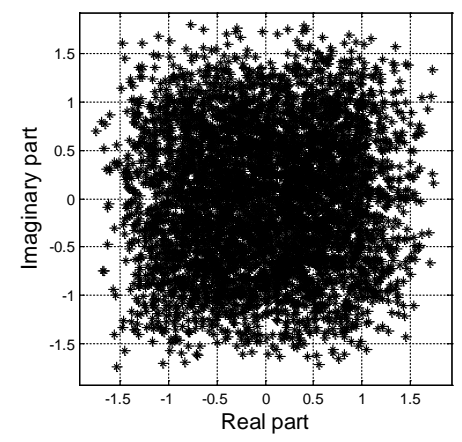

(b) Input constellations

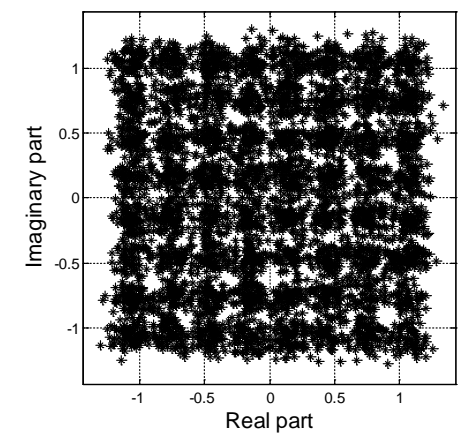

(c) Output of MMA

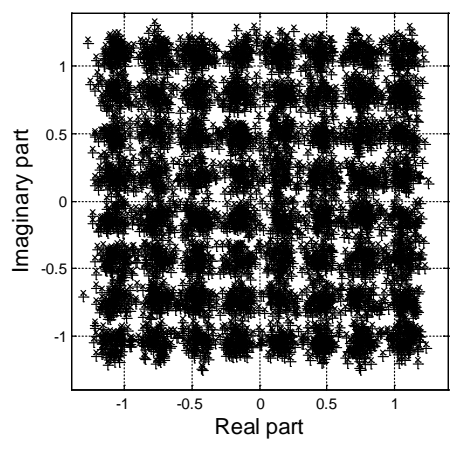

(d) Output of WTMMA

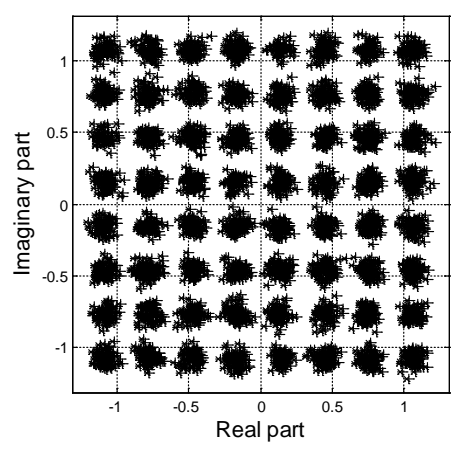

(e) Output of DNAGA-WTMMA

Fig.3. Simulation results

Figure 3 shows that the proposed algorithm has the fastest convergence rate and the smallest mean square error comparison with the MMA and WTMMA. Due to tabu search algorithm and the premature degree of population are introduced into DNA genetic algorithm, thus, the convergence rate is improved significantly and shortcoming of premature convergence also are overcame. Figure 3 shows that the constellation of the proposed algorithm is more compact and clear than other two algorithms.

\section{Conclusion}

In order to improve the performance of blind equalization algorithm, an orthogonal wavelet transform multi-modulus blind equalization algorithm based on tabu search DNA genetic optimization algorithm (DNAGA-WTMMA) was proposed. Tabu search algorithm was introduced to crossover operations to avoid circuitous search, ensure that the different paths can be searched, 
and escape from local optimum. The crossover probability based on the fitness value of population is adjusted to improve convergence rate significantly and reduce mean square error of WTMMA. Therefore, compared with the MMA and WTMMA, the proposed algorithm is more suitable for real-time signal processing in communication system.

\section{Acknowledgements}

Project supported the Major Project of Nature Science Foundation of Higher Education Institution of Jiangsu Province, China(Grant No.13KJA510001), Jiangsu Scientific Research Achievements in Industrialization Project, China(JHB 2012-9), and Jiangsu Province, the 2015 Annual General University Graduate Students Practice Innovation Program(SJLX15_0398).

\section{References}

[1] Jian Yang, Jean-Jacques Werner, Guy A Dumont. The Multimodulus Blind Equalization and Its Generalized Algorithms [J]. IEEE journal on selected areas in communications, 200220 (5) 997-1015.

[2] Vahid Faghihi, Kenneth F Reinschmidt, Julian H Kang. Construction Scheduling Using Genetic Algorithm Based On Building Information Model [J]. Expert Systems With Applications, 201441 (16) 7565-7578.

[3] Leonard M Adleman. Molecular computation of solutions to combinatorial problems[J]. Science, 1994266 (5187) 1021-1024.

[4] Yong-Jie Li, Jie Lei. A feasible solution to the beam-angle-optimization problem in radiotherapy planning with a DNA-based genetic algorithm [J]. IEEE Transactions on biomedical engineering, 201057 (3) 499-508.

[5] Fabio Bertequini Leao, Rodrigo A F Pereira, Jose R S Mantovani. Fast Fault Section Estimation In Distribution Control Centers Using Adaptive Genetic Algorithm[J]. International Journal of Electrical Power \& Energy Systems, 201463 (2014) 787-805.

[6] Duan Zhang, Yan-Ling Xia, Xiong-Xiong He, et al. Multi-step evolution strategy based DNA genetic algorithm for parameters estimating. The 4th International Conference on Intelligent Control and Information Processing (ICICIP)[C]. Beijing, 2013, 828-835.

[7] Tanumay Datta, N Srinidhi, A Chockalingam, et al. Random-Restart Reactive Tabu Search Algorithm for Detection in Large-MIMO Systems[J]. IEEE communications letters, 201014 (12) 1107-1109.

[8] Hao Gao, Sam Kwong, Bao-jie Fan, et al. A Hybrid Particle-Swarm Tabu Search Algorithm for Solving Job Shop Scheduling Problems[J]. IEEE transactions on industrial informatics, 201410 (4) 2044-2054.

[9] Ye-Cai Guo, Ying-Ge Han, Chao Yang. Orthogonal Wavelet Transform based Sign Decision Dual-mode blind equalization Algorithm. 9th International Conference on Signal Processing, ICSP[C]. Beijing, 2008, 80-83. 